\title{
Erratum to: A genome-wide association study of prostate cancer in West African men
}

Michael Blaise Cook $\cdot$ Zhaoming Wang $\cdot$ Edward D. Yeboah $\cdot$ Yao Tettey $\cdot$ Richard B. Biritwum $\cdot$ Andrew A. Adjei $\cdot$ Evelyn Tay $\cdot$ Ann Truelove $\cdot$ Shelley Niwa $\cdot$ Charles C. Chung $\cdot$ Annand P. Chokkalingam $\cdot$ Lisa W. Chu $\cdot$ Meredith Yeager · Amy Hutchinson · Kai Yu • Kristin A. Rand · Christopher A. Haiman • African Ancestry Prostate Cancer GWAS Consortium • Robert N. Hoover · Ann W. Hsing $\cdot$ Stephen J. Chanock

Published online: 3 December 2013

(C) Springer-Verlag Berlin Heidelberg 2013

\section{Erratum to: Hum Genet}

DOI 10.1007/s00439-013-1387-z

Regrettably, one of the author group African Ancestry Prostate Cancer GWAS Consortium has been wrongly cited in the published article. The correct author group order should read as
Michael Blaise Cook - Zhaoming Wang - Edward D. Yeboah • Yao Tettey $\bullet$ Richard B. Biritwum • Andrew A. Adjei $\bullet$ Evelyn Tay $\bullet$ Ann Truelove $\bullet$ Shelley Niwa $\bullet$ Charles C. Chung $\bullet$ Annand P. Chokkalingam $\bullet$ Lisa W. Chu $\bullet$ Meredith Yeager $\bullet$ Amy Hutchinson $\bullet$ Kai Yu $\bullet$ Kristin A. Rand $\bullet$ Christopher A. Haiman $\bullet$ African Ancestry Prostate Cancer GWAS Consortium • Robert N. Hoover • Ann W. Hsing • Stephen J. Chanock
The online version of the original article can be found under doi:10.1007/s00439-013-1387-z.

M. B. Cook $(\bowtie) \cdot$ Z. Wang · C. C. Chung $\cdot$ M. Yeager · A. Hutchinson $\cdot$ K. Yu $\cdot$ R. N. Hoover $\cdot$ S. J. Chanock Division of Cancer Epidemiology and Genetics, National Cancer Institute, NIH, DHHS, 9609 Medical Center Drive, Rm 7-E106, MSC 9774, Bethesda, MD 20892-9774, USA

e-mail: cookmich@mail.nih.gov; michael.cook@nih.gov

Z. Wang $\cdot$ M. Yeager $\cdot$ A. Hutchinson

Cancer Genomics Research Laboratory, NCI-DCEG,

SAIC-Frederick Inc, Frederick, MD, USA

E. D. Yeboah $\cdot$ Y. Tettey $\cdot$ R. B. Biritwum

A. A. Adjei - E. Tay

Korle Bu Teaching Hospital, PO Box 77, Accra, Ghana

E. D. Yeboah · Y. Tettey · R. B. Biritwum •

A. A. Adjei - E. Tay

University of Ghana Medical School, PO Box 4236,

Accra, Ghana

A. Truelove $\cdot$ S. Niwa

Westat, 1600 Research Boulevard, Rockville,

MD 20850-3129, USA

\author{
A. P. Chokkalingam $\cdot$ A. W. Hsing \\ School of Public Health, University of California, Berkeley, \\ CA, USA \\ L. W. Chu \\ Cancer Prevention Institute of California, 2201 Walnut Avenue, \\ Suite 300, Fremont, CA 94538, USA \\ K. A. Rand · C. A. Haiman \\ Department of Preventive Medicine, Norris Comprehensive \\ Cancer Center, Keck School of Medicine, University of Southern \\ California, Los Angeles, CA 90033, USA
}

A. W. Hsing

Stanford Cancer Institute, Stanford University, 875 Blake Wilbur

Drive Stanford, Stanford, CA 94305, USA 